\title{
Style, comfort and fuctionality
}

If you're looking for a fully flexible, state of the art treatment centre, the new entry level INTEGO Ambidextrous from Dentsply Sirona provides style, comfort and functionality to suit every practitioner. This latest fully functional treatment centre serves both rightand left-handed practitioners and features an outstanding ergonomic design and intelligent swivel mechanism, which means it can be effortlessly switched from right to left and

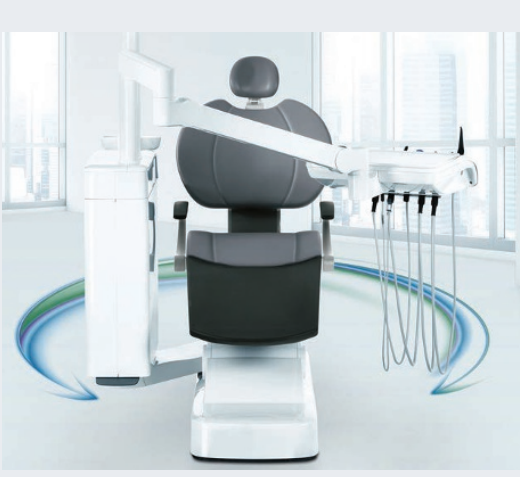
vice versa in just 15 seconds.

The swivel mechanism is extremely straightforward. A switch on the water unit is released by foot and moved around the chair, with the backrest moving automatically to the correct position.

The operating light, monitor and dentist elements are quickly repositioned, a process that can easily be carried out between appointments. No screws need to be released or secured, meaning no special training or other equipment is required. The intuitive and precise swivel movement minimises the risk of damage to hoses or collision with other components.

Designed with your budget in mind, there are four INTEGO Ambidextrous models available, offering outstanding value for money and Dentsply Sirona quality you can rely on.

Contact Dentsply Sirona on 08000723313 or visit dentsply.com/en-uk.

\section{The latest generation of 3D printing}

As a pioneer of CAD/CAM technology and specialist in dental processing techniques, BEGO is systematically developing its $3 \mathrm{D}$ printing system. The traditional family company presented the new smart Varseo S for labside $3 \mathrm{D}$ printing of restorations made from resins at the recent International Dental Show in Cologne. The extended VarseoWax material spectrum and the new BEGO Otoflash light-curing unit were shown as well. Validated processes and the associated process documentation provide security to the users to be 'MDR ready'.

The Varseo $S$ is characterised by its compact and stylish design and a larger building plate, which ensures the simultaneous printing of even more objects. Additionally, the network capability of the device permits rapid BEGO Service via remote control. The high building speed is independent of the number of elements to be produced. The unique cartridge system allows a material change in seconds, low material consumption and minimal material ageing.

In addition to the tested VarseoWax resins for the production of CAD/Cast partial denture frames, surgical guides, dental models and individual impression trays, BEGO presented the new resin VarseoWax Splint E for 3D printing of occlusal splints at IDS. In the course of 2017,
BEGO is going to expand its range of resins by providing VarseoWax Base for 3D printing of full denture bases as well as VarseoWax Temp for 3D printing of long-term temporaries.

The new light-curing unit BEGO Otoflash allows a rapid and reliable light-curing of VarseoWax resins.

The comprehensive 3D printing service package includes a qualified hotline support and online access to the BEGO 3D Academy, which provides the Varseo user with information on $3 \mathrm{D}$ printing via video tutorials, webinars, tips and tricks.

More detailed information on the BEGO 3D printing system Varseo $S$ can be found online at www.bego.com/3d-printing.

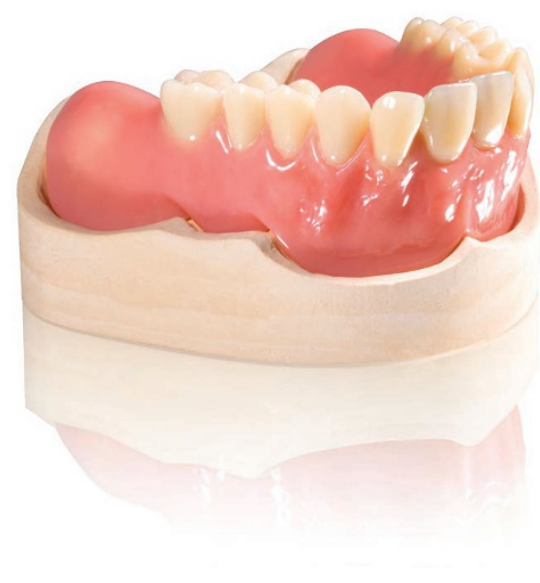

